\title{
Perceptions Towards Childhood Asthma and Barriers To Its Management Among Patients, Caregivers and Healthcare Providers: A Qualitative Study From Ethiopia
}

\section{Eden Kassa}

College of Health Sciences, Ethiopian Defence University

Rahel Argaw Kebede

School of Medicine, Addis Ababa University

Bruck Messele Habte ( $\nabla$ bruck.messele@aau.edu.et)

School of Pharmacy, Addis Ababa University

\section{Research Article}

Keywords: children with asthma, caregivers, barriers to management, qualitative study, Ethiopia

Posted Date: December 29th, 2021

DOI: https://doi.org/10.21203/rs.3.rs-1203028/v1

License: (c) (1) This work is licensed under a Creative Commons Attribution 4.0 International License.

Read Full License 


\section{Abstract \\ Background}

The management of asthma, which is one of the major causes of childhood morbidity and mortality has been affected by non-adherence to recommended treatment regimens with severe consequences. The aim of the present study was therefore to explore the perceptions of the children with asthma, their caregivers and their healthcare providers towards asthma and barriers to long term childhood asthma management in an institutional setting in Addis Ababa, Ethiopia.

\section{Methods}

A qualitative descriptive design was followed for the present study that used individual interviews as a data collection method. The study participants were 23 pairs of children with asthma that had treatment follow ups in two tertiary hospitals and their caregivers and eight healthcare providers who cared for these children. The data was analyzed using thematic analysis approach.

\section{Results}

The study findings revealed that the children and their caregivers were facing physical, emotional and social burdens related with asthma and reported low adherence to their recommended treatment regimens. Factors affecting childhood asthma management were found to be limited awareness about asthma and its management and inadequate education received from healthcare professionals. Nonadherence to especially inhaled corticosteroids appears to be influenced by necessity beliefs towards chronic administration of treatment regimens and concerns related with difficulty of administration, fear of side effects and general bad attitude towards it, in addition to their low availability and affordability.

\section{Conclusions}

Varied perceptions about asthma and its management were reported by children with asthma and their caregivers compared to the biomedical recommendation that were related to different factors. This in turn may contribute to the low adherence of the children to their recommended regimens and suboptimal health outcomes. The findings support the need for strong asthma care and education programs that are sensitive to local and individual patients' and family perceptions and experiences including emotional distress and of the need to institute chronic care approach.

\section{Background}

Asthma is one of the common respiratory diseases affecting the communities globally with prevalence ranging from $10-13 \%$ [1]. In the African region the prevalence of asthma especially in children less than 
15 years increased from $12.1 \%$ in 1990 to $13.9 \%$ over two decades. In sub-Saharan countries, estimated prevalence varies widely between countries: Ethiopia $9.1 \%$, Kenya $15.8 \%$, Nigeria $13.0 \%$, and South Africa $20.3 \%[2]$.

Asthma has been defined by the Global Initiative for Asthma as "a heterogeneous disease, usually characterized by chronic airway inflammation which is defined by the history of respiratory symptoms such as wheeze, shortness of breath, chest tightness and cough that vary over time and in intensity, together with variable expiratory airflow limitation" [3]. While asthma can develop at any stage in life, it most commonly develops in early childhood [4]. Its burden has been shown to excessively affect children and is said to be among the top ten chronic conditions for 5-14-year-olds. Asthma can be treated depending on the severity of the symptoms. According to the current Global Initiative for Asthma recommendations, the main goal of asthma treatment is to achieve and maintain good control of symptoms and inhaled corticosteroids (ICS) are recommended as one of the best maintenance therapies [3].

Non-adherence to asthma treatment is associated with poor asthma management, higher healthcare utilization, healthcare costs, and reductions in health-related quality of life [5]. In management of childhood asthma, the issue of adherence is particularly essential and challenging because they are easily vulnerable to airway restructuring which can lead to permanent life-threatening airway obstruction [6]. The findings from studies in the west have depicted how factors related to the children's and caregivers' health beliefs about asthma and its treatment and their poor relationships with healthcare providers (HCPs) undermine effective asthma management [7]. Such studies could guide the development of interventions. They however need to consider local contexts including local cultures and thus the rationale for this study. The intent of this study was therefore to explore the perception of children with asthma, their caregivers and HCPs towards asthma and its asthma management. The findings from this study can guide policy makers and HCPs in addressing issues raised in the management of asthma in children.

\section{Methods}

\section{Study design and settings}

The study followed a descriptive qualitative approach [8] to enable an in-depth understanding of the perceptions of the children and their caregivers towards asthma and its management which has received limited attention to the best of our knowledge especially in the present context. The two sites selected for the present study were Tikur Anbessa Specialized Hospital (TASH) and St. Paul's Hospital Millennium Medical College (SPHMMC), both public tertiary hospitals and located in Addis Ababa, Ethiopia. These hospitals run pulmonary follow up clinics led by pediatric pulmonary and critical care physicians and serve the largest pool of asthmatic children and thus the rationale for their selection as the study sites.

\section{Study participants' selection}


This study used purposive sampling technique to select children with asthma, their caregivers and HCPs including physicians, nurses and pharmacists as participants. This sampling technique was preferred to select study participants who could express their feeling independently and had more exposure with asthma and its barriers to treatment. The criteria used for children study participants and by their virtue for the caregivers were age of 8 to 15 years old, attending chest clinic follow up for at least six months, using ICS for at least three months and ability to communicate either in Amharic or English and not being severely ill. Furthermore, effort was made to include a heterogenous sample by including study participants (children with asthma and their caregivers) who had different residence localities and severity levels of asthma to provide a diverse range of cases relevant to the topic. The criteria for the physicians and nurses was working with children for at least 3 months in the chest clinic while for the pharmacists it was working in the pediatric pharmacy during the study period.

The children and their caregivers were approached on their follow up day, and given the information about the study. Upon their assent and consent respectively, they were appointed for interview. The selected children were paired with their caregivers but interviewed separately to capture their views without interference from their caregivers. Sampling and recruitment of participants had continued until saturation was achieved which was when "the major themes were identified and participants started expressing the same idea repeatedly" [9].

\section{Data collection}

Data collection took place from April to June 2018 using semi structured interview guides which were adaptations from similar studies and also based on Kleinman's explanatory model and Horne's necessity - concerns model to enable exploration of patients', caregivers' and HCPs perceptions towards asthma illness and its treatment including adherence to recommended regimens and any possible discrepancies in view of the above concepts among the different actors. The interview guides attempted to capture the culturally determined process of making sense of asthma, ascribing meanings to symptoms of asthma, understanding causal attributions, and expressing suitable expectations of treatment and related outcomes [10-13]. The interview guide for the children and their caregivers included questions on their perceptions of asthma, its perceived cause as well as the sign and symptoms, use of anti-asthma medications with focus on ICS, perceived necessity and concerns related with the medications, adherence to recommended regimens, perceived barriers to asthma management and relationship with HCPs (Additional file 1). The questions for the health care providers revolved around experiences with managing childhood asthma and medication-related counseling (Additional file 2).

The first author conducted the face-to-face interviews which ranged from 18 to 40 minutes at a convenient location such as the participant's home, separate office from the clinic and cafeteria inside both hospitals but far from the pediatric chest clinic which allowed privacy for the participants. All interviews were audio-recorded while observation field notes were taken to record the major points as well as the contextual and nonverbal conditions. Following the first few interviews, the collected data was transcribed and analyzed and discussions held with the second and last authors to guide further 
interviews. Furthermore, debriefings were carried out with these few participants to improve subsequent interviews.

\section{Data analysis}

The audio-recorded data as well as field notes were transcribed verbatim and then translated into English. The first author and another researcher with experience in qualitative research methods independently coded the sampled data and then discussed the coding to develop coding frames for each of the interviews. In this regard, Kleinman's explanatory model and the necessity-concerns model were used to define codes although active search was made to identify emerging codes. Then themes and subthemes were developed in the data based on the two models and other emerging ideas to describe patterns, relationships and trends between variables following the recommendations for descriptive qualitative analysis [8].

\section{Rigor and trustworthiness}

The rigor of the findings was enhanced by different means. One is the use of different sources such as children with asthma, their caregivers as well as their HCPs which enabled rich and triangulated findings. On the other hand, the investigators were a clinical pharmacist, a pediatric pulmonary and critical care physician and a social pharmacist with experience in medicines use study and qualitative research methods which allowed the issue to be viewed from different perspectives. The first author who was also the data collector went through a process of reflexivity from the initial stages right through the data collection, analysis and report writing to enhance the rigor of the study. Furthermore, three HCPs and two caregivers' study participants were given the chance to comment on their respective interview transcripts.

\section{Results}

\section{Participants' backgrounds}

A total of 54 participants took part in the study of which 46 were children and their family caregivers while the rest were the HCPs caring for the children. Majority of the children with asthma (15/23) and their caregivers were recruited from TASH. Of the total child participants, majority were female $(13 / 23)$, within the age range of 8 to $12(19 / 23)$ and between the grades of 1 and $5(14 / 23)$. With regards to the family caregiver participants, majority were Muslim, were married females, were in the age range of 35 to 45 and completed high school. It was also apparent that nine were patients with asthma as can be seen in Table 1. Among the eight HCPs, majority were females and physicians (Table 2).

\section{Themes}

The key findings from this explorative study were clustered into four key themes: perceptions towards asthma, burden of asthma, management of asthma and adherence to recommended treatment.

\section{Perception towards asthma}


Both children and their caregivers reported that they were not well informed about the meaning of asthma by the HCPs. Majority of children with asthma have described signs and symptoms of asthma as difficulty of breathing, coughing and sweating. Caregivers who were asthmatic themselves thought that genetic predisposition solely causes asthma. For example, one of them said that...

"In my opinion the cause is hereditary because I am also asthmatic." (Caregiver 8)

Some of the physicians also described that the children had limited knowledge about the disease and admitted that they didn't adequately work on creating awareness about the disease to the children and their caregivers which affected their medication taking behaviors.

"Majority of children with asthma and caregivers do not know much about the disease and its behavior...So, they discontinue the medication as soon as they get a relief and they come back when symptoms aggravate." (Physician 1)

All of the physicians also believed that majority of children with asthma as well as caregivers, had no clear knowledge about the cause of asthma as depicted by the following quote.

"Personally, I don't think that children with asthma and their caregivers have a clear knowledge about the cause and aggravating factor of asthma. For example, some of them believed that asthma can be transmitted through coughing and inhalation like Tuberculosis." (Physician 3) 
Table 1

Socio demographic characteristics of caregivers

\begin{tabular}{|c|c|}
\hline Characteristics & Number \\
\hline \multicolumn{2}{|l|}{ Sex } \\
\hline Female & 15 \\
\hline Male & 8 \\
\hline \multicolumn{2}{|l|}{ Marital status } \\
\hline Divorced & 4 \\
\hline Married & 18 \\
\hline Widowed & 1 \\
\hline \multicolumn{2}{|l|}{ Age group } \\
\hline $25-34$ & 8 \\
\hline $35-44$ & 12 \\
\hline $45-50$ & 3 \\
\hline \multicolumn{2}{|l|}{ Religion } \\
\hline Muslims & 14 \\
\hline Christian & 9 \\
\hline \multicolumn{2}{|l|}{ Level of education } \\
\hline Unable to read and write & 6 \\
\hline High school & 10 \\
\hline Higher education (first degree and above) & 7 \\
\hline \multicolumn{2}{|l|}{ Monthly income } \\
\hline$<1000$ & 3 \\
\hline $1000-3000$ & 15 \\
\hline$>3000$ & 5 \\
\hline \multicolumn{2}{|l|}{ Asthma profile } \\
\hline Asthmatic & 9 \\
\hline Non asthmatic & 14 \\
\hline
\end{tabular}


Almost all children with asthma expressed the psychological and physical burden of asthma on their daily life. For example, one of them described how it affected play time with friends.

"I usually feel its impact while I play with my friends. There were times when I stopped playing because I was afraid that I might suddenly face difficulty of breathing in the middle of the game." (Child 20)

Table 2

Profile of health care providers

\begin{tabular}{|ll|}
\hline Characteristics & No \\
\hline Profession & \\
\hline Physician & 4 \\
\hline Nurse & 2 \\
\hline Pharmacist & 2 \\
\hline Sex & \\
Female & 5 \\
Male & 3 \\
\hline
\end{tabular}

The children also explained that asthma made them feel worried and nervous. The emotional burden extends to worries related to the inability to administer the medicine by themselves. On the other hand, majority of caregivers reported that asthma caused feeling of dependency on the children. They also described that it made them shy and limited their communication with people. Fear of exacerbation of asthma was another thing both the children and caregivers worried as depicted by the quote below.

"My life has become different since I was diagnosed with asthma. Emotionally, I feel anxious in my daily activity." (Child 1)

"I am so worried about my child's condition in case she suddenly experiences exacerbation of asthma symptoms." (Caregiver 8)

\section{Management of asthma}

Both medical and non-medical approaches were described in the process of managing asthma. By and large, the biomedical approach was the mainstay of treatment as would be expected in this hospitalbased study. Majority of the children with asthma as well as their caregivers however cited that they used home remedies such as "tazma mar" (a special type of honey obtained from a type of bee which is stingless), and other remedies that they believed will help with their asthma such as 'milk with honey and garlic' as can be seen in the following quotes. 
"Sometimes, I will give him a special honey called "tazma mar" because, I heard that it is good for relieving cough and other symptoms of asthma." (Caregiver 3)

"Oftentimes, I discontinued administering the medication and started natural treatment like dressing him in a sweater, giving him honey and milk with garlic." (Caregiver 12)

There was also the practice of religious healing to help manage the asthma as can be seen in the quote below.

"We usually went to church to pray and I will drink holy water when my symptoms get worse." (Child 3)

All of the physicians admitted that they were not correctly following the recommendations of pediatric asthma management correctly and not achieving the goals of asthma treatment because of patient load in the hospitals. Additionally, some of the physicians seem to lack the requisite skills for managing childhood asthma as shown by the following quote.

"Personally, I am not fully confident when to prescribe steroids for children since the diagnosis is not clear and it is too difficult once it is started." (Physician 4)

\section{Adherence to recommended treatment}

With regard to medication taking behavior, majority of children and caregiver participants described how they didn't take them regularly as recommended and instead discontinued if they had no problem of breathing. They also described that they used one opened inhaler after three or four months.

"I am taking the 'oxygen' (ICS) whenever my symptoms aggravate, like when I have difficulty of breathing." (Child 3)

Majority of children and their caregivers believed that their asthma is under control while using ICS which they describe as 'oxygen'. Both participant groups appreciated the necessity of the ICS although its use was in the form of a reliever rather than as a controller medicine as depicted below.

"I think it is a better medicine because it will enable me to breathe when I get difficulty of breathing. I mean it gives me oxygen." (Child 8)

The health care providers likewise explained as to how children with asthma were taking ICS for some time and would then discontinue as soon as they felt better. Some of the HCPs also reported that children considered ICS as a reliever medicine instead of the preventer medicine as it was intended, as can be seen in the following quote.

"Most of children with asthma were not taking ICS as we prescribed; they took it on a PRN (on need) basis by themselves." (Physician 2)

The children and caregiver participants reported different concerns about the medicine that are related to its 'bad' taste and smell, and loss of smell and taste. In addition, they also reported difficulty of 
administration, fear of side effects and general dislikes that contributed to their decisions not to take it as illustrated by the following quotes.

"I don't like its smell. In addition, my tongue will not sense anything for some time after I administer the ICS." (Child 3)

"As I heard from my friends, ICS will cause some side effects like hypertension and I think it will cause my child to be dependent only on this medication." (Caregiver 22)

Some caregivers also described that they faced some challenges from the society about their children's asthma therapy as depicted by the following quote from one of the caregivers.

"My neighbors and even my wife tried to persuade me that I shouldn't administer ICS to my child as they thought it will make her dependent on this medication. However, this didn't make me change my mind..." (Caregiver 9)

All of the physicians believed that children with asthma did not take their prescribed medicines, especially the ICS, as recommended. They explained that the reasons may be bad perception about the medicine, fear of side effects and 'creating adaptation' and difficulty of administration.

"The main concern is that majority of the caregivers had a bad perception about the ICS... For example, they thought the medicine will make adaptation and through time the patient will not respond to this medicine..." (Physician 2)

Furthermore, all participants including the health care providers complained about the unavailability of ICS and other essential medicines in the hospitals as depicted below by an emotional mother who cried while discussing this and an HCP.

"I was so disappointed when I couldn't find the medication when my child was very sick. I thought the hospital has a separate chest clinic unit and serves many children with asthma daily. So, it was hard to believe that this huge hospital lacked this medication." (Caregiver 21)

\section{Interaction with healthcare providers}

The interaction that the children and caregivers had with the HCPs can be described in generally as good and friendly. The high patient load in these tertiary hospitals however preclude the provision of adequate education about asthma. Some of the HCPs also expressed expectations that the children may have some knowledge about their condition given by the other colleagues which further reduced the amount of information provided as depicted by the following quote.

"Since I believe that the doctors will advise them before coming to the pharmacy, I don't usually advice about the disease. However, I will tell them little about the administration of ICS." (Pharmacist 1)

\section{Discussion}


The study revealed that the child participants and their caregivers expressed different explanatory models about asthma and its management. Furthermore, they reported experiences of emotional, physical and social burdens in relation with asthma in their daily activities. It was also apparent that both the children and caregivers had limited awareness of the recommended biomedical regimens especially the ICS. This may have led to the reported adherence problems which in turn led to symptom aggravation. In this regard, the apparently low necessity beliefs to take the ICS continuously and concerns about the side effects as well as the availability and affordability issues seem to have negatively affected adherence to their recommended regimen.

The emotional, physical and social burden faced by both the children and their caregivers seems to have impacted the family's social life and affected the children so that they feel shy and dependent having limited communication with others. Similar findings were reported by a similar study done among British South Asians and white caregivers where they experienced social and emotional impacts as a result of their children's asthma [14]. Another study done among Hong Kong Chinese caregivers also reported how they lost confidence and felt powerless due to repeated hospital admissions of their children [15]. Such phenomena are bound to negatively affect the treatment outcomes and the well-being of both the children and their caregivers. Policy makers and concerned HCPs should therefore give due attention to these burdens and strive to facilitate psychological as well as social support for the child patients as well as their caregivers.

All groups of participants have described adherence issues in implementing recommended treatment regimens related to the ICS, including its use as a reliever medication. This may have to do with limited awareness about its necessity but also concerns including about its side effects, 'creating adaptation' and loss of taste and smell. Similar observations are reported in studies done among whites as well as non-white communities with the concerns and adherence issues pronounced among the minority populations $[7,14,15]$. This variation could be partly attributed to cultural differences among the different communities which in turn could affect the trust towards the biomedical regimens. This calls for HCPs to provide culturally adapted patient and caregiver education sessions and involve these caregivers as asthma educators to enhance peer to peer support.

It was also apparent from the study findings that the management of childhood asthma was suboptimal and unsatisfying even for the HCPs which obviously needed revamping. One approach is setting up an asthma clinic that can be run by trained nurses such as the one reported by participants in a British study. These participants reported how they were able to access a regular asthma review session which also included additional support on inhaler techniques and peak flow meter use [14]. Experiences with nurseled care are also reported in the resource limited settings of sub-Saharan Africa where an intervention study involving a nurse-led management program for asthma with the close support of physicians in Cameroon led to marked improvement in patient outcomes and was well received by the community [16]. While there are no reported studies of a similar experience in Ethiopia, the experience with HIV care and treatment can be leveraged to improve patient care for NCDs such as pediatric asthma through the chronic care model [17]. In the tertiary settings of the present study, nurse or pharmacist educators can be 
assigned following these models to provide requisite chronic care for these patients as part of the chest clinic. These providers can also be trained to assess for psychosocial issues so as to refer to appropriate section in the hospital.

Another major concern had to do with access to anti-asthmatic medications especially the low availability of the ICS in both hospitals. Irrespective of the diverse manner of use of this group of medicines, the unavailability in these public hospitals could have detrimental impacts on the children's life. The low availability of ICS is supported by a wider study that assessed the availability, pricing and affordability of beclomethasone, budesonide and salbutamol in 52 selected low- and middle-income countries including Ethiopia. The findings of this study revealed the low availability of these medicines with the figure for beclomethasone standing at $19 \%$ in public hospitals and a bit higher at $42 \%$ in private outlets. Nevertheless, these medicines were largely unaffordable to the majority of the population in a low income setting such as Ethiopia [18]. This calls for yet another policy intervention to improve the supply and financing of these medicines in the public sector and the community at large. The full implementation of the community and social health insurance scheme would be a good step in this regard [19].

\section{Strength And Limitation Of Study}

The study is the first in its kind in the country as well as in its triangulation technique which incorporated the data sets including children with asthma, caregivers and HCPs view point. However, the transferability of the findings may be limited to tertiary settings as the contexts for patients at primary health care tiers and private setups may be different. The present study was done in hospital setup where patients or care givers are to a certain extent informed about asthma during their follow up. So, in depth community interviews can additionally help to understand asthma perception and its management in the community to further elicit the cultural impact on the perception of asthma.

\section{Conclusions}

Children with asthma and their caregivers expressed different perceptions about asthma and its management compared to the biomedical recommendation and reported facing physical and emotional and social burdens. Major concerns related with ICS were difficulty of administration, fear of side effect and general bad attitude towards it. These and other factors such as the low availability and affordability of the ICS could have contributed to the low adherence of the children to their recommended regimens. This in turn may contribute to suboptimal health outcomes.

Therefore, there is a need to work to increase the awareness of the patients and their caregivers towards asthma and the treatment including ICS and work on its adherence in children in concordance with care givers and HCPs. This may call for the development of a chronic care model that can address not only a culturally adapted asthma education but also provide or facilitate psychosocial support for both the children with asthma and their caregivers. The medicines access issue is another issue that needs to be 
addressed including through hastening the community and social health insurance programs that are under different stages of implementation.

\section{Abbreviations}

HCPs: Healthcare providers; ICS: Inhaled corticosteroids; SPHMMC: Saint Paul's Hospital Millennium Medical College; TASH: Tikur Anbessa Specialized Hospital

\section{Declarations}

\section{Acknowledgements}

The authors would like to acknowledge all the children, caregivers and healthcare providers who participated in this study. The authors would also like to acknowledge the two study hospitals and the respective administrators for their facilitation of the study. Lastly, the authors acknowledge the support of Mr. Fisseha Shiferie for his support during the coding of the interview data.

\section{Ethical approval and consent to participate}

Ethical approval was obtained from Research Ethics Committee of the School of Pharmacy, Addis Ababa University (ERB/SOP/11/10/2018) that was followed by permissions from the administrators of the two hospitals prior to starting data collection. Informed assents and consents were obtained from all participants before the data collection. Furthermore, measures were taken to ensure privacy during the interviews, and confidentiality and anonymity once the data were collected and during the analysis and reporting. In addition, the information that was collected in this research will be kept confidential in a locked computer where nobody, other than the PI, can access it.

\section{Consent for publication}

Not applicable

\section{Availability of data and materials}

The datasets used and/or analyzed during the current study are available from the corresponding author on reasonable request.

\section{Competing interest}

"The authors declare that they have no competing interests"

\section{Funding}

The source of funding which was part of the graduate study of the first author was Addis Ababa University. 


\section{Authors' contribution}

EK: conceptualization, methodology, investigation, analysis, writing (drafting, review and editing); RA: analysis and writing (reviewing and editing); BMH: methodology, analysis, writing (review and editing). All authors read and approved the final submission.

\section{Authors' information}

${ }^{1}$ College of Health Sciences, Defense University, Bishoftu, Ethiopia.

2 Department of Pharmaceutics and Social Pharmacy, School of Pharmacy, College of Health Sciences, Addis Ababa University, Ethiopia.

${ }^{3}$ Department of Pediatrics and Child Health, School of Medicine, College of Health Sciences, Addis Ababa University, Ethiopia

\section{References}

1. Lawson JA, Janssen I, Bruner MW, Hossain A, Pickett W. Asthma incidence and risk factors in a national longitudinal sample of adolescent Canadians: A prospective cohort study. BMC Pulm Med. 2014;14.

2. Adeloye $D$, Chan KY, Rudan I, Campbell H. An estimate of asthma prevalence in Africa: A systematic analysis. Croat Med J. 2013;54:519-31.

3. Global Initiative for Asthma. Global Strategy for Asthma Management and Prevention. 2021;:1-217. https://ginasthma.org/wp-content/uploads/2021/05/GINA-Main-Report-2021-V2WMS.pdf\%0Ahttps://ginasthma.org/gina-reports/.

4. Asher I, Pearce N. Global burden of asthma among children. Int J Tuberc Lung Dis. 2014;18:126978.

5. Mäkelä MJ, Backer V, Hedegaard M, Larsson K. Adherence to inhaled therapies, health outcomes and costs in patients with asthma and COPD. Respir Med. 2013;107:1481-90.

6. Bender B, Wamboldt F, O'Connor SL, Rand C, Szefler S, Milgrom H, et al. Measurement of children's asthma medication adherence by self report, mother report, canister weight, and Doser CT. Ann Allergy, Asthma Immunol. 2000;85:416-21.

7. Callery P, Milnes L, Verduyn C, Couriel J. Qualitative study of young people's and parents' beliefs about childhood asthma. Br J Gen Pract. 2003;53:185-90.

8. Doyle L, McCabe C, Keogh B, Brady A, McCann M. An overview of the qualitative descriptive design within nursing research. J Res Nurs. 2020;25:443-55.

9. Creswell JW. Educational research: Planning, conducting, and evaluating quantitative and qualitative research. 4th edition. Boston: Pearson; 2012. 
10. Kleinman A, Eisenberg L, Good B. Clinical Lessons from Anthropologic and Cross-Cultural Research. Ann Intern Med. 1978;88:251-8.

11. Petrie KJ, Weinman JA. Perceptions of health and illness: Current research and applications. Amsterdam: Harwood Academic Publishers; 1997.

12. Peláez S, Lamontagne AJ, Collin J, Gauthier A, Grad RM, Blais L, et al. Patients' perspective of barriers and facilitators to taking long-term controller medication for asthma: A novel taxonomy. BMC Pulm Med. 2015;15:1-11. doi:10.1186/s12890-015-0044-9.

13. Searle A, Jago R, Henderson J, Turner KM. Children's, parents' and health professionals' views on the management of childhood asthma: A qualitative study. npj Prim Care Respir Med. 2017;27:1-6.

14. Lakhanpaul M, Culley L, Robertson N, Bird D, Hudson N, Johal N, et al. A qualitative study to identify parents' perceptions of and barriers to asthma management in children from South Asian and White British families. BMC Pulm Med. 2017;17:1-12.

15. Chong YY, Leung D, Mak YW. When control exacerbates distress: A qualitative study exploring the experiences of Hong Kong chinese parents in caring for a child with asthma. Int J Environ Res Public Health. 2018;15.

16. Kengne AP, Sobngwi E, Fezeu LL, Awah PK, Dongmo S, Mbanya JC. Nurse-led care for asthma at primary level in rural sub-Saharan Africa: The experience of Bafut in Cameroon. J Asthma. 2008;45:437-43.

17. Letebo M, Shiferaw F. Adapting HIV patient and program monitoring tools for chronic noncommunicable diseases in Ethiopia. Global Health. 2016;12:26. doi:10.1186/s12992-016-0163-y.

18. Babar ZUD, Lessing C, Mace C, Bissell K. The availability, pricing and affordability of three essential asthma medicines in 52 low- and middle-income countries. Pharmacoeconomics. 2013;31:1063-82.

19. Tefera BB, Kibret MA, Molla YB, Kassie G, Hailemichael A, Abate T, et al. The interaction of healthcare service quality and community-based health insurance in Ethiopia. PLoS One. 2021;16 8 August:116. doi:10.1371/journal.pone.0256132.

\section{Supplementary Files}

This is a list of supplementary files associated with this preprint. Click to download.

- Additionalfile1.docx

- Additionalfile2.docx 